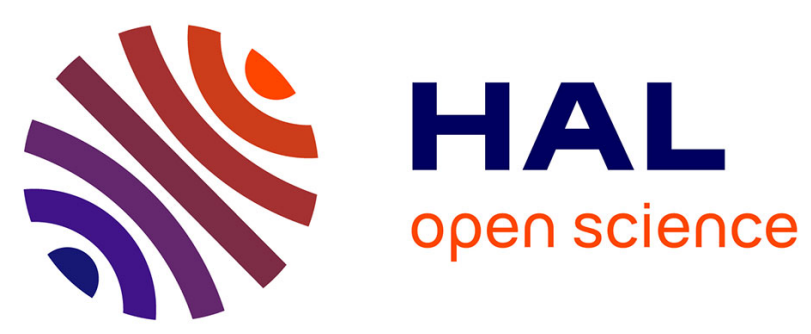

\title{
Units in the primary auditory cortex of the Japanese monkey can demonstrate a conversion of temporal and place pitch in the central auditory system
} H. Riquimaroux, T. Hashikawa

\section{- To cite this version:}

H. Riquimaroux, T. Hashikawa. Units in the primary auditory cortex of the Japanese monkey can demonstrate a conversion of temporal and place pitch in the central auditory system. Journal de Physique IV Proceedings, 1994, 04 (C5), pp.C5-419-C5-425. 10.1051/jp4:1994588 . jpa-00253083

HAL Id: jpa-00253083

https://hal.science/jpa-00253083

Submitted on 1 Jan 1994

HAL is a multi-disciplinary open access archive for the deposit and dissemination of scientific research documents, whether they are published or not. The documents may come from teaching and research institutions in France or abroad, or from public or private research centers.
L'archive ouverte pluridisciplinaire HAL, est destinée au dépôt et à la diffusion de documents scientifiques de niveau recherche, publiés ou non, émanant des établissements d'enseignement et de recherche français ou étrangers, des laboratoires publics ou privés. 


\title{
Units in the primary auditory cortex of the Japanese monkey can demonstrate a conversion of temporal and place pitch in the central auditory system
}

\section{H. RIQUIMAROUX and T. HASHIKAWA}

Neural Systems Laboratory, Frontier Research Program, The Institute of Physical and Chemical Research (RIKEN), Wako, Saitama 351-01, Japan

\begin{abstract}
The present study has examined whether the temporal pitch known as the "missing fundamental" is co-place-coded with the place pitch in the auditory cortex, using adult Japanese monkeys (Macaca fuscata) prepared for chronic recordings. The stimuli were white noise bursts, tone bursts and bursts of a combination of higher harmonics of a low frequency. Unit recordings were made from the contralateral (left) primary auditory cortex (AI). During recording sessions, the animal was anesthetized with a mixture of nitrous oxide and oxygen, supplemented by ketamine and xylazine injections. The data have confirmed a tonotopicity, low frequency anteriorly while high frequency posteriorly, in AI when the recording time window was short $(<20$ msec) with stimulus at threshold levels. For longer time windows and/or suprathreshold stimulus levels, determining the best frequency (BF) for a neuron was often difficult. In the low frequency area $(\leq 500 \mathrm{~Hz})$, the same neuron responded to $\mathrm{BF}$ as well as a combination of successive higher harmonics of $\mathrm{BF}$ without $\mathrm{BF}$. However, they did not or little responded to the harmonics themselves. The findings imply that the temporally-coded pitch at the periphery appears to be already place-coded together with the place pitch by the same neuron at AI. This evidence agrees well with previous psychoacoustical findings.
\end{abstract}

\section{INTRODUCTION}

Since 19th century, it is a well known fact in psychoacoustics that the ear sometimes perceives tones which do not correspond to any of the Fourier-components of the objective sound $[1,2]$. When we add several higher harmonics, we have a pitch sensation of the low-frequency fundamental which does not exist in the sound (called the "missing fundamental" or "virtual pitch", Fig. 1). Although the phase-locked firing corresponding to the virtual pitch exists in the temporal discharge pattern of the cochlear nerve fibers [3], it has been believed that the missing fundamental pitch is extracted not by the peripheral but by the central auditory system [4]. The missing fundamental pitch sensation cannot be masked by a low-pass noise. If it is originated in the cochlea, it should be masked by the low-pass noise. The missing fundamental sensation can be created by a dichotic presentation of harmonics from each ear [5]. If it is 
created by the peripheral auditory system, the dichotic stimulation with harmonics would not produce the missing fundamental pitch. However, little is neurophysiologically investigated about this problem. This study examined whether the place pitch (fundamental frequency, fo) and the temporal pitch (missing fundamental) are co-place-coded by the same neuron at the level of primary auditory cortex (AI).

\section{METHODS}

\subsection{Subjects}

Adult Japanese monkeys (Macaca fuscata) were used for this study. After an otoscopic examination, they were screened with the auditory brainstem responses (ABR) and slow cortical responses (SVR) before surgical procedure. A monkey with poor ABR or SVR was rejected from the experiment.

\subsection{Surgical procedure}

All surgeries were aseptically performed with sufficient anesthesia by means of an intravenous injection of a mixture of ketamine and xylazine. After cleaning the skull, a metal post was glued at the vertex. A temporal portion of the skull was removed in round shape over which a chamber (diameter $=3 \mathrm{~cm}$ ) was placed for chronic recordings. The dura remained intact. Recordings started 2 weeks after the surgery. Intramuscular injections of antibiotics for 7 days and topical application of antibiotics to the surgically wounded area prevented infections.
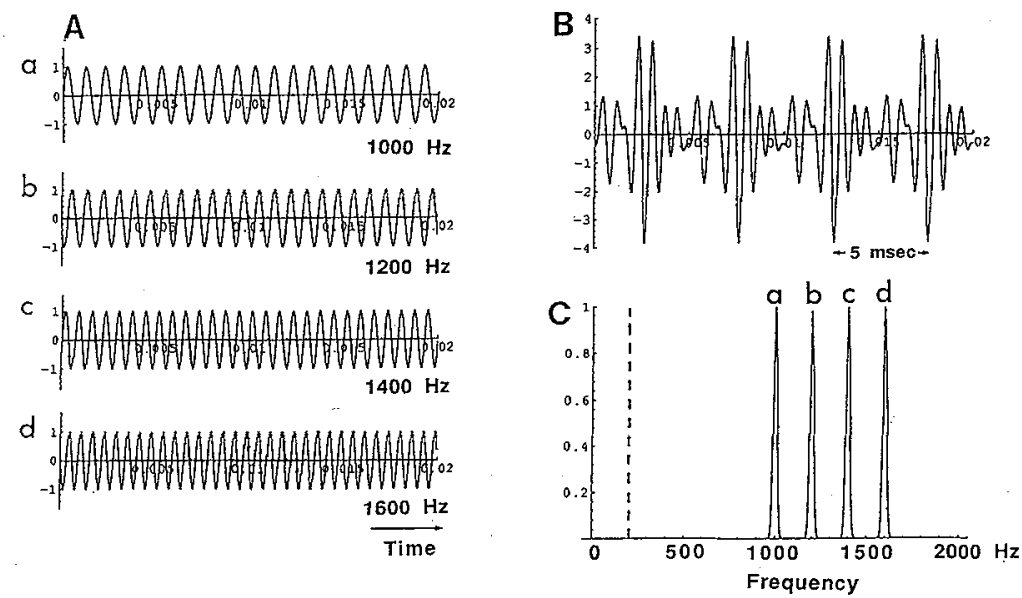

Fig. 1 A combination of four higher harmonics to produce the "missing fundamental". A: $1000,1200,1400$ and $1600 \mathrm{~Hz}$ sinusoidal signals (pure tones) are illustrated in a, b, $c$ and $d$, respectively. B: Temporal wave pattern produced by adding $1000,1200,1400$ and $1600 \mathrm{~Hz}$. A periodicity of $5 \mathrm{~ms}$, corresponding to $200 \mathrm{~Hz}$, is visible. C: Power spectrum of the synthesized wave shown in B. Peaks for $1000,1200,1400$ and $1600 \mathrm{~Hz}$ can be observed but no peak for the missing fundamental $(200 \mathrm{~Hz}$, broken line). 


\subsection{Stimulus}

The stimuli used were white noise bursts, tone bursts and bursts of a combination of higher harmonics of a low fundamental frequency (fo). White noise was digitally generated by a random noise generator (NF,WG-721A). Sinusoidal waves were also digitally generated by an arbitrary waveform generator (Yokogawa, AG-1200). Continuous white noise and sinusoidal waves were passed through a tone-burst modulator (DIA Medical, DPS-721) to make burst stimuli. The stimulus was attenuated (Tamagawa, TPA-308A), amplified (Sony, TA-N7050) and then presented by a loud speaker to the animal's right ear which was located $25 \mathrm{~cm}$ from the speaker. The left external ear canal was occluded by an ear plug. The sound stimulus was monitored through a microphone located at the place where the animal's ear was placed with a measuring amplifier (ACO, 6030) and an FFT analyzer (Advantest, R9211B). Rise/fall time was $10 \mathrm{msec}$ and the plateau was fixed at $20 \mathrm{msec}$, making total duration of 40 msec. The stimulus was presented once every $3 \mathrm{sec}$. Frequency varied from $100 \mathrm{~Hz}$ to 25 $\mathrm{kHz}$. The stimulus intensity was changed from the neuron's threshold to $70 \mathrm{~dB}$ SPL for tone bursts and to $90 \mathrm{~dB}$ SPL for white noise bursts. The sound pressure level was measured with continuous tones and white noise because the RMS meter could not follow the envelope of burst stimuli. The white noise burst was used to search auditory units.

\subsection{Recordings}

The animal was put on a stage in an electromagnetically shielded sound proof room. The head was immobilized by fixing the metal post on the skull to a hollowed metal rod with a screw. Unit recordings were made with glass-coated Elgiloy electrodes from the left primary auditory cortex (AI) on the superior temporal plane (Fig. 2A). During recording sessions, the animal was anesthetized with a mixture of nitrous oxide and oxygen, supplemented by ketamine and xylazine injections. Body temperature was maintained at $38^{\circ} \mathrm{C}$. The heart rate was monitored (Nihonkoden, AC-611G) throughout the experiment. The electrode was tilted by $55^{\circ}$ from the horizontal plane and orthogonal to the anterior-posterior line. The electrode was advanced by a pulse-motor micromanipulator (Narishige, custom-made). Cortical activities were filtered ( $300 \mathrm{~Hz}-3$ $\mathrm{kHz}$ ), amplified (Nihonkoden, $\mathrm{AB}-651 \mathrm{~J}$ ) and fed into a window discriminator (DIA Medical, DSE-435) to produce a pulse corresponding to a neural action potential, which was sent to a computer (NEC, PC-9801DA) to generate PST (post-stimulus-time) histograms on line. Twenty trials were usually summed to create one PST histogram. The PST histogram was triggered $30 \mathrm{msec}$ prior to the stimulus onset and continued for 350 msec (Fig. 3). The minimum bin width of the PST histogram was 1 msec. Recordings were made once a week for each animal. After each recording session, the chamber was cleaned and sealed for the next experiment. 


\subsection{Neuroanatomy}

After finishing all the recordings, the animal was sacrificed by a lethal dose of pentobarbital, followed by a perfusion through the heart with saline and then $4 \%$ paraformaldehyde in phosphate buffer. Immediately after the perfusion, the brain was taken out and immersed in a sucrose solution. Nissl and parvalbumin stainings were made with $30 \mu \mathrm{m}$ sections, in which the primary auditory cortex was identified as the koniocortex [6].Then, the recording sites were examined. Parvalbumin staining shows AI [7].

\section{RESULIS}

We confirmed that our recordings were made from AI by comparing a Nissl staining containing electrode tracks with a parvalbumin staining indicating AI (Fig. 2). The darkly stained area of within the lateral sulcus (LS) in Fig. 2c indicates AI [7]. Tonotopicity was found in antero-posterior direction. Low frequency neurons $(\leq 500 \mathrm{~Hz}$,
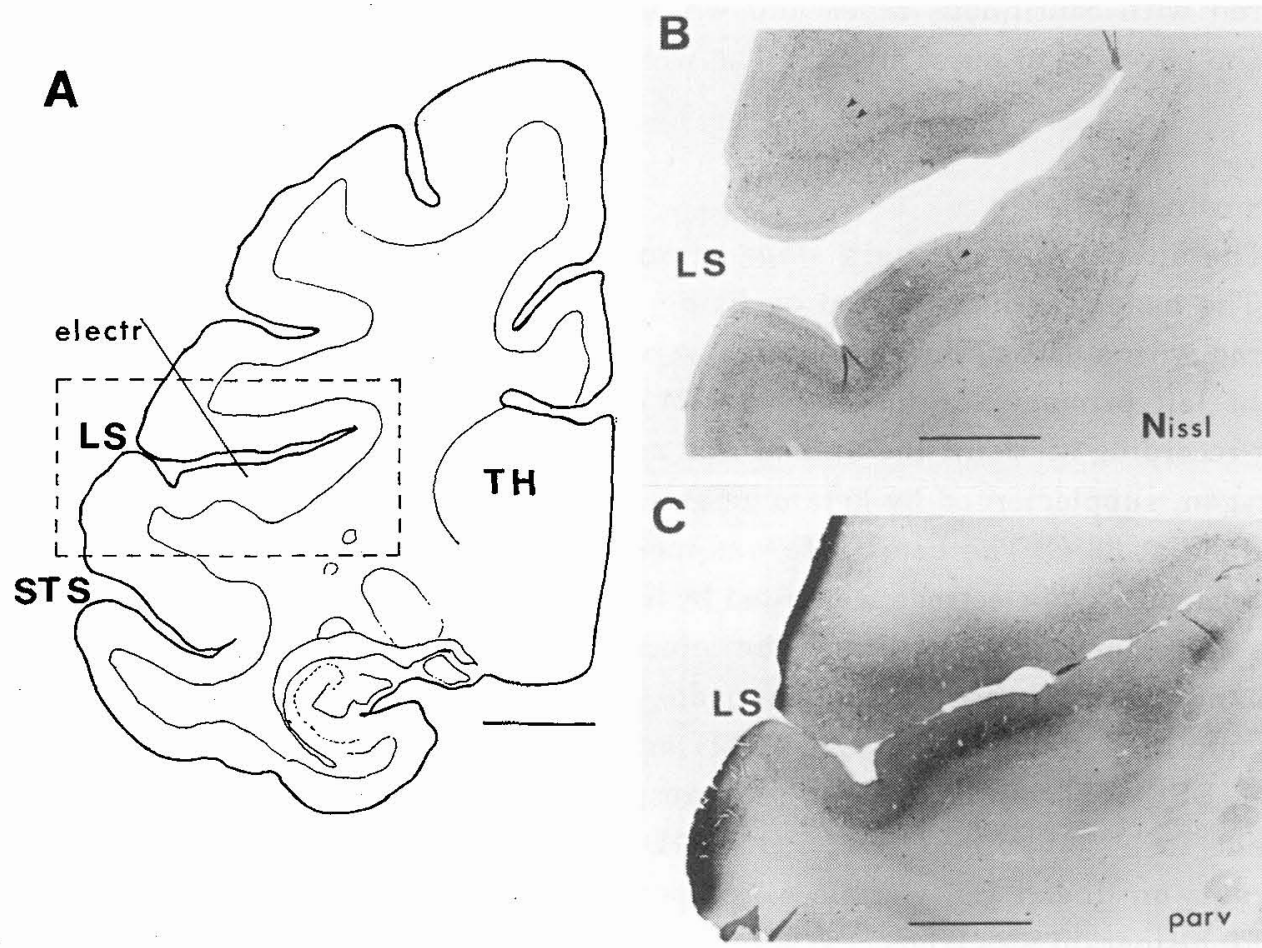

Fig. 2 Frontal sections showing recording sites. A: A schematic electrode track is illustrated. electr: electrode, LS: lateral sulcus, STS: superior temporal sulcus, TH: thalamus. B: Nissl staining of a recording site (outlined by broken line in A) showing electrode tracks (arrow head). C: Parvalbumin staining of a recording site (outlined by broken line in A). Darkly stained area indicates the primary auditory cortex. Bars indicate $5 \mathrm{~mm}$ in A and 3 $\mathrm{mm}$ in $\mathrm{B}$ and $\mathrm{C}$. 


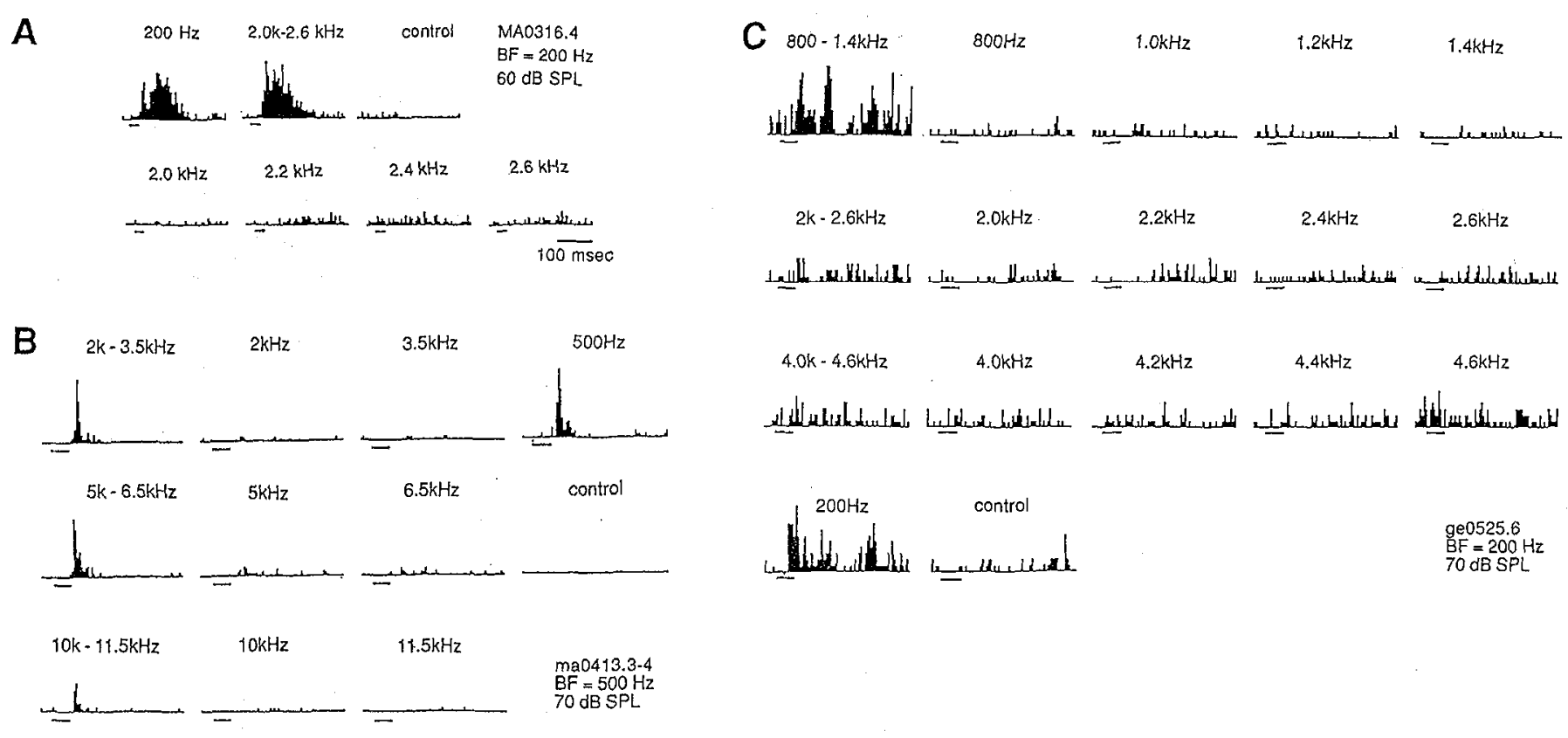

Fig. 3 Typical responses of low frequency neurons to the fundamental frequency (fo), the combination of higher harmonics without the fo and each higher harmonic component. A, top row: from left responses to $200 \mathrm{~Hz}$, a complex of $2.0 \mathrm{k}+2.2 \mathrm{k}+2.4 \mathrm{k}+2.6 \mathrm{kHz}$ and control condition, respectively, bottom row: responses to $2.0 \mathrm{kHz}, 2.2 \mathrm{kHz}, 2.4 \mathrm{kHz}$ and $2.6 \mathrm{kHz}$, respectively. $\mathrm{BF}=200 \mathrm{~Hz}$, Intensity $=60 \mathrm{~dB}$ SPL (each frequency component of the complex sound is $48 \mathrm{~dB} \mathrm{SPL}$ ). B, top row: responses to a complex of $2.0 \mathrm{k}+2.5 \mathrm{k}+3.0 \mathrm{k}+3.5 \mathrm{kHz}, 2.0 \mathrm{kHz}, 3.5 \mathrm{kHz}$ and $500 \mathrm{~Hz}$, respectively. middle row: responses to a complex of $5.0 \mathrm{k}+5.5 \mathrm{k}+6.0 \mathrm{k}+6.5 \mathrm{kHz}, 5.0 \mathrm{kHz}, 6.5 \mathrm{kHz}$ and control condition, respectively. bottom row: responses to a complex of $10.0 \mathrm{k}+10.5 \mathrm{k}+11.0 \mathrm{k}+11.5 \mathrm{kHz}, 10.0 \mathrm{kHz}$ and $11.5 \mathrm{kHz}$, respectively. $\mathrm{BF}=500 \mathrm{~Hz}$, Intensity $=70 \mathrm{~dB}$ SPL (each frequency component of the complex sound is $58 \mathrm{~dB}$ SPL). C, first row: responses to a complex of $800+1.0 \mathrm{k}+1.2 \mathrm{k}+1.4 \mathrm{kHz}, 800 \mathrm{~Hz}, 1.0 \mathrm{kHz}, 1.2 \mathrm{kHz}$ and 1.4 $\mathrm{kHz}$, respectively. second row: responses to a complex of $2.0 \mathrm{k}+2.2 \mathrm{k}+2.4 \mathrm{k}+2.6 \mathrm{kHz}, 2.0 \mathrm{kHz}, 2.2 \mathrm{kHz}, 2.4 \mathrm{kHz}$ and $2.6 \mathrm{kHz}$, respectively. third row: responses to a complex of $4.0 \mathrm{k}+4.2 \mathrm{k}+4.4 \mathrm{k}+4.6 \mathrm{kHz}, 4.0 \mathrm{kHz}, 4.2 \mathrm{kHz}, 4.4 \mathrm{kHz}$ and $4.6 \mathrm{kHz}$, respectively. fourth row: response to $200 \mathrm{~Hz}$ and control condition. $\mathrm{BF}=200 \mathrm{~Hz}$, Intensity $=70 \mathrm{~dB}$ SPL (each frequency component of the complex sound is $58 \mathrm{~dB} \mathrm{SPL}$ ). 
located in the anterior part of AI, were characterized to examine the conversion of the place pitch and the temporal pitch produced by a combination of four higher harmonics without the fo (Fig. 1). All of 15 neurons examined responded to the fo as well as a combination of higher harmonics without the fo. However, they did not or little responded to each higher harmonic component (Fig. 3ABC). Moreover, they only responded to a combination of higher harmonics in a certain frequency range (Fig. 3BC).

\section{SUMMARY and DISCUSSION}

In the low frequency area $(\leq 500 \mathrm{~Hz})$, a single neuron responds both to the best frequency (BF) and to a combination of successive higher harmonics which creates a temporal periodicity identical to the BF (Fig. 1), but not to these higher harmonic components themselves (Fig. 3ABC). Further, the higher harmonics have to be within a certain frequency range for the combined harmonics to produce the same pitch as the fo (Fig. 3BC). Thus, not only the periodicity created by the harmonics but also the actual frequency of the harmonics appear to be important for the missing fundamental pitch extraction. This tendency is similar to previous human psychoacoustical findings [1, 2, 8] (Fig. 4). For example, Fig. 4 indicates that human subjects can perceive the missing fundamental pitch of $100 \mathrm{~Hz}$ only when the lowest harmonic frequency is between $500 \mathrm{~Hz}$ and $2 \mathrm{kHz}$ [8]. Human lesion studies have also shown data agree with the present findings. Namely, patients without AI have difficulties to perceive the missing fundamental pitch but that patients with temporal lobectomy with an intact AI show a normal missing fundamental sensation [9, 10]. A recent auditory-evoked magnetic field study has implied that a combination of higher harmonics without the fo and the fo are processed in the same area in the auditory cortex of humans [11], which also agrees with the present results. Schwarz and Tomlinson [12] failed find neurons in the auditory cortex of the rhesus monkey which responded to the fo as well as the combination of higher harmonics without the fo. The reason why they could not find

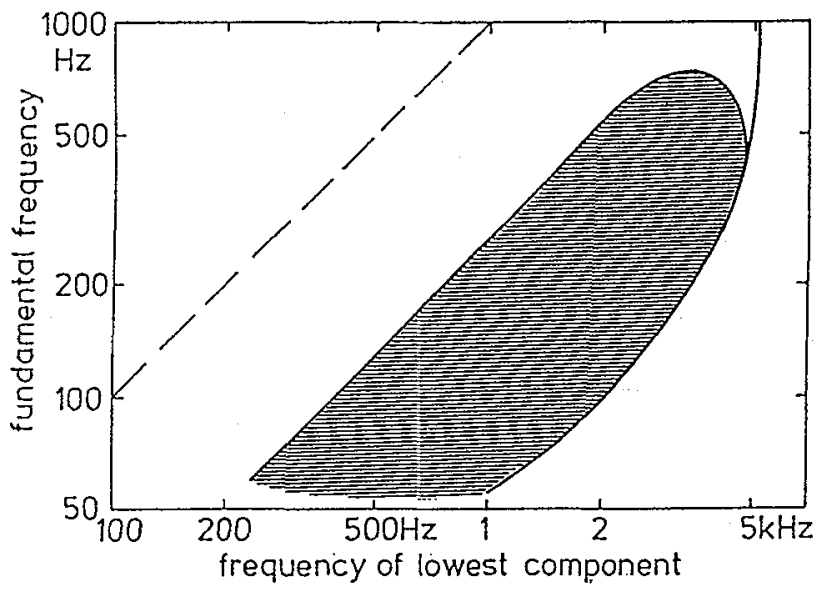

Fig. 4 Region where the missing fundamental pitch exists. Fundamental frequency as a function of the lowest component in the higher harmonic complex. Shaded area: region where the missing fundamental exists. Broken line: fundamental frequency. (from Zwicker and Fastl, 1990) 
such neurons, which we have described here, is not clear. The present data suggest that the temporally-coded pitch in the periphery appears to be already place-coded together with the place pitch by the same neuron to produce an identical pitch at AI. Experiments with a low-pass masker with the missing fundamental situation, a dichotic presentation of harmonics from each ear and an inharmonic combination of frequency components with a constant spacing frequency will be interesting tests to examine the central processing of the missing fundamental pitch extraction.

\section{acknowledgement}

The authors show gratitude to Dr. E. G. Jones for his help to conduct this study. The research was supported by Frontier Research Program, RIKEN.

\section{Reference}

1) Seebeck, A. (1841) Beobachtungen über einige bedingungen der entstehung von tönen. Ann. Phys. Chem. 53: 417-436.

2) Schouten, J. F. (1938) The perception of subjective tones. K. ned. akad. Wet. Proc. 41: 1086-1093.

3) Delgutte, B. (1980) Representation of speech-like sound in the discharge pattern of auditory-nerve fibers. J. Acoust. Soc. Am. 68: 843-857.

4) Moore, B. C. J. (1989) An Introduction to the Psychology of Hearing. Academic Press, London, pp 158-193.

5) Houtsma, a. J. M. and Goldstein, J. L. (1972) The central origin of the pitch of complex tones: Evidence from musical interval recognition. J. Acoust. Soc. Am. 51: 520-529.

6) Morel, A., Garraghty, P. E. and Kaas, J. H. (1993) Tonotopic organization, architectonic fields, and connections of auditory cortex in macaque monkeys. $J$. Comp. Neurol. 335: 437-459.

7) Wallace, M. N., Kitzes, L. M. and Jones, E. G. (1991) Chemoarchitecture organization of the cat primary auditory cortex. Exp. Brain Res. 86: 518-526.

8) Zwicker, E. and Fastl, H. (1990) Psychoacoustics. Springer-Verlag, Berlin.

9) Zatorre. R. J. (1988) Pitch perception of complex tones and human temporal-lobe function. J. Acoust. Soc. Am. 84: 566-572.

10) Bharcha, J. J., Tramo, M. J. and Zattore, R. J. (1993) Abstraction of the missing fundamental following bilateral lesions of auditory cortex. Soc. Neurosci. Abstr. 19. 1687.

11) Pantev, C., Hoke, M., Lütkenhöner, B. and Lehnertz, K. (1989) Tonotopic organization of the auditory cortex: Pitch versus frequency representation. Science 246: $486-488$.

12) Schwarz, D. W. F. and Tomlinson, R. W. W. (1990) Spectral response patterns of auditory cortex neurons to harmonic complex tones in alert monkey (Macaca mulatta). J. Neurophysiol. 64: 282-298. 